\title{
CAPACITACIÓN EN AREA RURAL QUE ES BÁSICA PARA UNA PRODUCTIVIDAD BENEFICIOSA
}

\section{GTRAINING IN RURAL AREA THAT IS PRIMARY FOR BENEFICIAL PRODUCTIVITY}

\author{
${ }^{1}$ Tello, Demetrio y ${ }^{2}$ Tello, Lily.
}

\begin{abstract}
Resumen
En las zonas rurales del país existe gran número de pequeños agricultores quienes no tienen adecuados conocimientos por falta de capacitación. Esto impide, a una población significativa, que sus directivos u otros puedan convertirse en empresarios y no lo consiguen por no haber podido superar obstáculos como: calidad de productos, conocimiento de estimación de la demanda futura para regularizar su producción, precios ofertados por los compradores, competencia, etc.; la suma de estas y otras dificultades son las que les crean problemas que no pueden solucionar.

Fue necesario la información divulgada sobre capacitación realizada por diversos medios y sus comentarios así como material: computadora, impresora, útiles de escritorio y como material de campo arena para vivero, fertilizantes, biosidas, nematicidas, bolsas de polietileno, tijeras, podadoras; en administración detalle de los insumos, sus importes, los costos unitarios así como los totales y otros.Los resultados de la capacitación, donde se han efectuado los talleres los encargados han comprobado que ha sido beneficiosa para los agricultores participantes, lo que permite vislumbrar que un reforzamiento instructivo en un periodo breve incrementará la productividad y mejorará sus ingresos.
\end{abstract}

Palabras clave: beneficios, capacitación, eficiencia, productividad.

\begin{abstract}
In rural areas of the country there is a large number of small farmers who do not have adequate knowledge for lack of training, because they have never had training until now. This prevents a significant population, their leaders become entrepreneurs and fail for not being able to overcome obstacles such as product quality, knowledge of estimating future demand to regularize their production, prices offered by buyers, competition, etc. the sum of these and other difficulties cause problems they cannot solve.It required about training information disclosed by different media and their comments and materials like computer, printer, stationery and material as sand field nursery , fertilizers, biocides, nematicides, polyethylene bags, scissors, clippers; detailed management of inputs, their amounts, unit and total costs, others. In places that have received training has been shown that the results are beneficial to the participating farmers. These allow instructive glimpse reinforcement in short increase productivity and improve their income. Results of training, which have been carried out workshops makers, have found that has been beneficial to the participating farmers, allowing a glimpse of instructional reinforcement in a short increase productivity and improve their income. Key words: profitability, training, efficiency, productivity.
\end{abstract}

\section{Introducción}

En el sector rural existe gran número de propietarios, que como negocios tienen, de acuerdo con nuestras normas tributarias, la categoría de micro o pequeñas empresas dedicadas, en este caso, a la producción agraria en general.

Vivimos en un país que tiene particularidades culturales, demográficas, geográficas y ecológicas, pero a pesar de esas dificultades se puede incrementar el área agrícola. Para lograrlo debe tenerse en cuenta previamente, la diversidad de climas, la calidad de los suelos, entre otros, los cuales permiten el cultivo de gran variedad de productos en costa, sierra y montaña.

Las actividades en esas parcelas, son realizadas, en su mayor parte, en propiedades cuya dimensión no excede de cinco hectáreas, es una pequeña área resultante de la distribución, por herencia, de ancestros, la cual usan como medio de subsistencia.

La proyección, para esos agricultores, debe ser diversificar la actividad agraria familiar en la cual, si se aplica tecnología en un periodo no muy amplio se modificaría el modelo actual que es de subsistencia, donde cultivan productos que atiende necesidades de la localidad, mientras que si incrementan la producción podrían atender al mercado nacional con perspectivas de ingresar al internacional.

La situación existe porque no se encuentran organizados, tienen escasa capacitación en tecnología productiva y herramientas de gestión empresarial; si conocieran ello posibilitaría, que pequeños productores generaran 
condiciones apropiadas para superar problemas de productividad así como de colocación de productos en los mercados interno y externo con precios justos; la excesiva atomización de las parcelas encarece el acceso a insumos, dificulta la adopción de tecnologías, limita acceso al crédito, resta poder de negociación frente a los comercializadores, dando lugar a que les ofrezcan precios más bajos por sus productos.

Para tal efecto es necesario implementar, como una necesidad, la capacitación en producción agraria así como en conocimientos empresariales propiciando la participación de los vinculados de la zona que son micro y pequeños empresarios con sus familiares y allegados si los hubiere.

El 2014 ha sido denominado el "Año Internacional de la Agricultura Familiar”- AIAF (2014). El Perú es el lugar de origen de una civilización que realizó el mayor número de progresos en agricultura en la antigüedad, domesticaron más de 180 especies las cuales han sido de utilidad para los seres humanos. Actualmente más del $94 \%$ de pequeños productores son quienes abastecen el $70 \%$ de la alimentación nacional, algunos de ellos continúan aplicando el conocimiento ancestral (Agronoticias no.396 Enero 2014).

Este trabajo enfatiza, como prioridad, la capacitación de empresarios de micro y pequeñas empresas, incluyendo familiares y relacionados, la misma que debe partir de lo básico y, por etapas, continuarla afianzando en el tiempo; el comienzo debe basarse en que, con sus propios recursos, mejoren su situación.

La información del IV Censo Agropecuario (2012), presenta como resultado que los pequeños agricultores (tenencia de una a cinco hectáreas) son 1,754.000 los cuales representan el 79\% del total de participantes del sector y en función de tierras disponibles son 269.000 hectáreas.

Una enseñanza, direccionada a sus necesidades, es primordial para que la resultante permita obtener una producción eficientey como consecuencia una venta mayor de productos y así conseguir más beneficios económicos; para ello es necesario que la instrucción inicial se concentre en actividades vinculadas directamente a sus quehaceres cotidianos. Lo que debe primar es adiestrarlos para que solucionen problemas urgentes, de lograrse es posible que, otros propietarios cercanos o allegados, al apreciar los resultados favorables, se adhieran a la instrucción, que en sí, es la preparación en las destrezas necesarias y de esa forma puedan enfrentar, con mayor seguridad, problemas simples y, de ahí, a los más complejos con ello, lo más probable es que los resultados se multipliquen y lo ideal es que se convierta en una tendencia, generando un avance paulatino, que traerá aparejado un progreso en su calidad de vida, mayores ingresos económicos, mejora en la productividad, resultado positivo en comercialización y otros.

En estas zonas las aplicaciones tecnológicas avanzadas son mínimas, lo más común es que esos conocimientos no les son informados y tampoco es usual aplicación de herramientas de administración; por ese motivo realizan sus transacciones en forma incipiente. Ese desconocimiento es la mayor desventaja cuando deben efectuar acuerdos con acopiadores o transar con financiadores, quienes adquieren su producción a precios mínimos, por ello, después de cubrir sus costos el diferencial que obtienen es tan insignificante que no les permite avanzar y en otros casos, en lugar de incrementar, disminuyen sus posibilidades.

Esta situación se mantiene desde hace bastante tiempo sin que se vislumbre cambio, modificación o mejora que tenga como finalidad conseguir un beneficio para el componente familiar; ello da como resultado que la pobreza sea parte de la vida cotidiana, lo cual desemboca, la mayor parte de veces, en hacer trabajar a los hijos (para disminuir costos) y que a sus vez redunda en no acceder a una educación mínima por eso, para ellos el futuro es totalmente incierto.

$\mathrm{Si}$ se toman en cuenta las condiciones actuales, en las cuales desarrollan las acciones productivas y empresariales las micro y pequeñas empresas se puede entender; que la situación vigente no les soluciona las dificultades crematísticas y estas tienden a ser cada vez más acuciantes. Adicionalmente lo común es que realicen sus transacciones, en forma individual, es el procedimiento, pero eso disminuye su capacidad de negociación por ello sus resultados son desfavorables, como no fomentan formar agrupaciones deviene en que sus costos (adquisiciones individuales y menores) sean onerosas y sus ingresos bajos, por el poco volumen (oferta individual), no tienen capacidad de negociación para la fijación de precios en los productos que deben enviar al mercado antes de que se malogren.

Para tratar de solucionar esas desventajas deben propender a la asociatividad en alguna de sus formas, las agrupaciones tienen importancia crucial debido a que, esa unión permite el incremento del área productiva lo cual conlleva un aumento en la producción, si se lograra esa situación, entonces les permitirá negociar mayores volúmenes tanto en las compras (salidas de dinero) como en las ventas (ingresos de dinero)

Una forma de revertir la situación es que propietarios $\mathrm{y}$ familiares tengan habilidades suficientes con lo que obtengan gradualmente en técnicas de producción así como las herramientas de administración $\mathrm{y}$, con su aplicación, comprueben los resultados cuantitativos y cualitativos, lo cual se reflejará en una mejor calidad de vida.

Si existe progreso en forma paulatina pero constante, en los pequeños agricultores se iniciará un cambio positivo en su situación real; asumiendo que entienden que saber aplicar técnicas en producción, herramientas administrativas en los procesos de comercialización y el acceso a informaciones vinculadas a sus productos (semillas, fertilizantes, precios, etc.) repercutirá en el desarrollo personal y social así como tener ingresos 
mayores a los actuales que son de supervivencia, esa posibilidad de crecimiento no la vislumbran en futuro cercano; al aplicar nuevos conocimientos se presentará una reversión de los resultados en los cuales se encuentran inmersos desde hace bastante tiempo.

El objetivo fue comprobar los beneficios de capacitar a empresarios, familiares y allegados relacionados con el sector rural, de micro y pequeñas empresas, en técnicas de producción como: componentes de suelo, calidad de semillas, disponibilidad de recursos hídricos, fertilizantes, etc., y en el caso de herramientas de administración, capacitación en: planificación de producción, oferta de productos, demanda de artículos, competencia, mercados, costos, financiación, personal, contabilidad, almacenes y otros. Por ello son necesarias varias materias por divulgar. Los resultados serán beneficiosos, indicando solo algunos donde se ha probado como: caso de Celendín (paltas) y caso de Moyobamba con (cacao).

\section{Materiales y métodos}

Los materiales necesarios fueron computadora, impresora, grabadora, USB, disco de archivo adicional, coordinaciones por internet, coordinaciones telefónicas, útiles de escritorio, tesis Cacao de pre grado de Cuellar D, T 2013 A. J para titularse de Ingeniero en Gestión Empresarial y de pos grado Human A. J 2013 tesis de palto para obtener el grado de $\mathrm{Mg} \mathrm{Sc}$ en Agronegocios, ambas investigaciones son de la Universidad Nacional Agraria La Molina, las cuales tienen como, parte de las actividades, las evaluaciones realizadas en diversos lugares del país donde se aplicaron acciones de capacitación en áreas agraria y de administración; para mencionar solo el caso del Palto, Provincia de Celendín, en la Región Cajamarca, lo referido a capacitación; se efectuaron, en diferentes fechas, a los miembros de la comunidad: seis talleres de capacitación en "Producción y técnicas de cultivos" y cuatro talleres de capacitación en "Comercialización del cultivo del palto" con resultados favorables, independientemente de otras actividades puntuales directas.

El acopio de información, para este trabajo, se realizó desde Agosto del 2013 hasta Marzo del 2014, principalmente en la Universidad Nacional Agraria La Molina, con información de tesis, textos, periódicos y revistas referidos al tema tratado

Se empleó el método "descriptivo- explicativo" debido que con la descripción se limitan las características del grupo y el "longitudinal retrospectivo" por qué la educación, mencionada en las tesis, se realizó con anterioridad a una campaña y se esperó su final para poder evidenciar los resultados favorables o desfavorables

\section{Análisis y discusión}

Obtener rendimientos altos sin adecuado uso de recursos hídricos, sin empleo de abonos que mejor se adecúen, sin evaluación del suelo, es bastante difícil, aún así no es fácil conseguir financiación estatal o particular; al no existir apoyo financiero la técnica por aplicarse debe basarse en lo que tienen. Tendiendo a tener resultados beneficiosos, si lo positivo se convierte en una tendencia se irá creciendo, en forma progresiva, avance pequeño al inicio, pero al ser recurrente tendrá, como correlato, un progreso que con el tiempo llegará a ser significativo, lo que servirá para la reversión de la situación que han tenido los pequeños productores durante muchos años (Lacki P, 2006).

De acuerdo con las ubicaciones geográficas de los pequeños negocios rurales es necesario que la capacitación se planifique, acorde con la necesidad específica, por ser diversos los productos, diferentes los suelos, variados los sistemas de riego, distinta la formación que tienen los responsables de las parcelas etc.; esto aparentemente determinará, en cada materia, una variedad de tópicos pero con adecuación las materias generales se puedan aplicar en lugares específicos con casi la certeza que los resultados serán provechosos. Por lo mencionado se deduce que capacitar es un potencial que aún no se aplica con intensidad y por ello no se ha podido beneficiar a los pobladores de sector rural.

Castañeda P 2013, expresa que para capacitación deben utilizarse módulos demostrativos y considera que sería lo más adecuado que los participantes estén conformados por agrupaciones de 20 a 30 familias.

Se considera que la capacitación se debe realizar separándola por niveles o etapas para que se efectúe en forma gradual.

Primer nivel, la información a transmitir es básica y directa como planificación anual de lo que deben cultivar, el conocimiento de los cultivos más acordes a la calidad del suelo que tienen, las enfermedades o plagas más comunes y así otros que posiblemente conocen pero agregando explicación del motivo por el cual deben realizarlo, es enterarlos, no solo de cómo se hace, sino porqué se realiza, qué beneficios posibles se obtendrán.

Segundo nivel, quienes participaron en el primer nivel y lo están aplicando serán los participantes. Este nivel comprende aspectos técnicos como, rotación de cultivos, eliminación o disminución de mermas o pérdidas, obtención de recursos hídricos, etc.

Tercer nivel, es más avanzado para quienes tienen conocimiento de técnicas de producción, están informados de la forma de participar en los mercados nacionales e internacionales, así como dar valor agregado a sus productos y otros.

Las áreas donde se debe incidir el adiestramiento son: producción y comercialización para que los administradores de las micro y pequeñas empresas mejoren en sus transacciones, al efecto se deben preparar programas para las áreas de capacitación con temas sencillos para que los interesados comprendan aspectos simples relacionados con la planificación, con los factores de la producción, conocimiento de lo que contienen los 
inventarios, lo imprescindible de la calidad, entre otros.

Medina M. F 2013, plantea tres interrogantes que son constantes en el área rural que crean limitaciones, las cuales son:

1. ¿Cuál es el principal obstáculo de crecimiento que enfrentan micro y pequeñas empresas?

2. ¿Qué estrategias de calidad se podrían implementar en el actual sistema de comercialización en micro y pequeñas empresas?

3. ¿Existen estrategias definidas a nivel comercial que permitan identificar mercados potenciales para incrementar las ventas en micro y pequeñas empresas? Para una racional instrucción deberán conocerse los problemas, evaluarlos y en base a ello, planificar las actividades por realizar, partiendo de análisis como los siguientes:

a) Cuáles son los mayores inconvenientes que tienen en sus actividades estableciendo orden de prioridad para obtener posibles soluciones y constaten que son las más acordes con las circunstancias que se les presentan.

b) Que materias, técnicas, métodos, productos, etc. son los que necesitan para evitar los inconvenientes que vienen afrontando, explicar las circunstancias por las cuales existen los problemas, como enfrentarlos, cuál será el resultado posible si se continúa y cuál es el beneficio estimado con los resultados de la capacitación.

c) Si hay conocimiento de aspectos productivos, comerciales, empresariales que están aplicando con relativo éxito en otras parcelas, hacerles comprender que si tratan y logran aplicarlas también resultarán beneficiados.

Webb R 2013, hace énfasis en el éxito de la pequeña agricultura del país debido a que, en los últimos 20 años el crecimiento en los pequeños fundos ha sido significativo, el incremento en promedio es $5.5 \%$ al año en productos como papa y maíz amiláceo duro $\mathrm{y}$, en otros cultivos, en promedio el crecimiento fue de $4 \%$ mencionado en este porcentaje a la yuca, la cebolla, el pallar y la arveja; realiza la precisión expresando que es uno de los éxitos más saltantes de la historia mundial e inclusive se hace mención que el crecimiento se presenta aún con las condiciones existentes del terreno y la tecnología empleada. Por ello se deduce que, con un entrenamiento a estos productores, el aumento aún puede continuarse presentando por algunos periodos más.

Empresario es la persona capaz de percibir oportunidades y elegir la mejor decisión para cumplir los objetivos, utilizando recursos: naturales, financieros, tecnológicos y humanos, para incrementar el valor de la empresa, es un proceso de liderazgo en el cual aplica dinero, tiempo y conocimientos sin descuidar la responsabilidad social (Varela, 2008).

Con la capacitación se tiende a que comprendan, apliquen o dispongan de las características empresariales, los cuales serán factores motivacionales, que enumeramos brevemente de un detalle sobre "competencias empresariales", que son: Visión de la carrera empresarial, Sensibilidad social, Autoconfianza, Pensamiento conceptual, Orientación al mercado, Toma de decisiones, Gestión de empresa, Orientación a la acción.

Las materias por desarrollar, de acuerdo a cada necesidad, son diversas, por ello solo se acompañan algunas, en relación al agro y la administración, presentados en forma genérica para que puedan ser utilizados en múltiples casos. Es de entendimiento que los contenidos son genéricos, pero debe considerarse, como base, para los casos específicos que necesitan los pequeños empresarios rurales.

\section{Información para capacitación en el área agraria}

Son múltiples los cursos teórico prácticos que se pueden dar teniendo en cuenta que existen diversos factores como clima, suelos, cultivo, hombre. Debido a la presencia de la Cordillera de los Andes en el Perú tenemos el 70\% de climas del mundo y su variabilidad hace que tengamos diversidad de suelos por ser el clima uno de los factores de formación de suelos. Esta variabilidad de clima y diversidad de suelos contribuye a que tengamos diferentes nichos ecológicos por lo que podemos tener innumerables cultivos así como múltiples variedades para un solo cultivo por ejemplo existen más de 3000 variedades para el cultivo de papa. Finalmente pero no menos importante, tenemos la acción del hombre que podemos traducirlo en tecnología y es ahí donde este trabajo de investigación se enfoca para aplicar en tres etapas, desde pequeños cambios sin inversión hasta mayores cambios con una inversión recuperable en el corto o mediano plaza con el objetivo de incrementar la cosecha comercial, mejorar la calidad del producto y por ende tener un mayor ingreso. En líneas generales, podemos citar algunos cursos los cuales pueden y deben adaptarse a cada parcela agrícola.

\section{Curso: Utilización y/o Preparación de abonos orgánicos}

Toda planta, incluso malezas, capta energía solar, extraen nutrientes del suelo y los tiene en su organismo; por lo tanto, son considerados abono verde si se cortan y dejan y/o incorporan al suelo; las más conocidas y utilizadas son las leguminosas. También se tienen residuos de origen animal como estiércol, orines, entre otros, que en nuestras condiciones aún se aplican directamente al campo lo cual no sucede en otros países debido a los malos olores que emanan y de las moscas que atraen. Otra opción, actualmente la más aceptada a nivel mundial es la mezcla de ambos residuos vegetales y animales para la preparación del compost (De Lucía, 2013).

Etapa 1:Luego de la cosecha, queda gran parte de restos vegetales los cuales pueden ser reciclados utilizándolos para devolver al suelo parte de los nutrientes extraídos por las plantas. Es importante asegurarnos que estos restos 
vegetales están libres de enfermedades o plagas y luego pueden ser picados e incorporados al suelo al momento de la preparación del campo. En época de fuertes lluvias, estos restos vegetales pueden utilizarse como mulch (Bagum, 2013), es decir, se colocan sobre el suelo sin necesidad de picarlos con el objetivo de proteger al suelo y disminuir el efecto del golpe de la gota de lluvia.

Etapa 2:Es reconocido el efecto de un abono orgánico obtenido de la mezcla de restos vegetales con excrementos animales más su respectiva preparación, el compost. Para poder llegar a esta etapa se requiere manejar temperatura de la mezcla y darle una aireación a la preparación, con el objetivo de que las temperaturas no sobrepasen los 70 grados Celsius y así proteger a los organismos benéficos. A su vez aseguramos no existan problemas de plagas y/o enfermedades provenientes de restos vegetales y/o excrementos (De Lucía, 2013).

Etapa 3:Una vez que se gana experiencia de la etapa anterior, se puede ver la producción de compost como un ingreso extra ya que se podría abastecer a otros agricultores del producto al ver las ventajas de su uso tanto en el recupero económico, mayor rendimiento del cultivo y en el mejoramiento del suelo en sus propiedades físicas, químicas y biológicas. Aquí se les puede explicar a los asistentes que la calidad de cada producto está en función al material que se utilice para producir compost; por ejemplo no es igual utilizar estiércol de caballo que estiércol de vaca, a su vez, no es igual estiércol de vacuno para engorde que vacuno lechero; además depende de la alimentación y agua que toman los animales. Así se tienen innumerables ejemplos que se pueden aplicar a cada condición geográfica en que nos encontremos y a cada parcela en particular en función a los materiales de la zona (Naeth, A; Wilkinson, S; 2013). En esta etapa podríamos avanzar y comenzar a trabajar con crianza de lombrices de tierra que pueden ser incorporadas al suelo y/u obtener el humus de lombriz, que es otro abono orgánico tan bueno como el compost y de mayor valor económico.

\section{Curso: Manejo Integrado de Plagas}

El Manejo Integrado de Plagas consiste en realizar una serie de acciones físicas, mecánicas, biológicas, genéticas, culturales y químicas para controlar, no erradicar, la sobrepoblación de insectos evitando que lleguen a convertirse en plaga. Se debe realizar la prevención, observación y aplicación.

Etapa 1:Dar a conocer que cada cultivo tiene sus plagas en función al clima, suelo y etapa de desarrollo vegetativo. El incremento de los insectos hasta convertirse en plagas se da porque tienen condiciones adecuadas para su crecimiento y desarrollo; por ello, es importante conocer el ciclo de vida de las plagas potenciales a presentarse y así estar preparados para poder combatirlas.
Etapa 2:En esta etapa aplicamos lo aprendido para que las condiciones de desarrollo de las plagas no sean las adecuadas. Por ejemplo, antes de sembrar podemos adecuar la densidad de siembra para disminuir la presencia de plagas difíciles de manejar, es así que si la plaga desarrolla bien en alta temperatura podemos disminuir la densidad de siembra para que exista una mayor aireación disminuyendo la temperatura; si la plaga requiere poca humedad podemos programar los riegos para que sean más frecuentes y de menor intensidad de tal manera que el suelo este húmedo por mayor tiempo. Un caso particular, como los áfidos presentes en la mayoría de los cultivos, son insectos que colocan sus huevos en el envés de hojas jóvenes por tanto se podría esparcir una mezcla de aceite con cebolla, huacatay y ajos para dificultar la colocación de huevos y el fuerte olor los ahuyenta. Se sabe que por plagas las cosechas pueden disminuir hasta un $70 \%$ por tanto cualquier control que realicemos contribuirá a que este alto porcentaje disminuya.

Etapa 3:En esta etapa podemos invertir en control de plagas pues se ha demostrado el efecto de recuperación de la inversión. Por ejemplo, conociendo el comportamiento nocturno de las plagas y además de lo realizado en la etapa anterior, podemos atraerlas con plásticos de colores llamativos que a su vez estén cubiertas con material pegajoso de tal manera que resultan ser trampas mortales. Si el cultivo es rentable podemos invertir en comprar insectos benéficos $\mathrm{y} / \mathrm{o}$ feromonas, todo ello en un intento de realizar un control no químico de las plagas. Finalmente llegado el caso que se requiera la aplicación de un químico este debe ser específico para la plaga tomando en cuenta al cultivo y así evitar dañar a organismos benéficos.

\section{Información para capacitación en el área administrativa}

\section{Comercialización}

Conociendo que la información a obtenerse es de terceros debe establecerse previamente una normatividad referida a responsabilidades del personal en las actividades que serán algunas o todas de las siguientes: Identificar al que está a cargo de organizar las tareas, conocer al que asignará los recursos y quién es el encargado de la distribución, señalar al de las actividades y finalmente al encargado de comprobar que se ejecuten las estrategias que permitirán la fluidez en las operaciones. Todo ello permitirá que lo que se produce sea comercializado, por ello en forma breve, se indica en que se debe capacitar.

Es conveniente disponer de un planteamiento, resultante de un análisis previo, que permita conocer la situación de la pequeña o micro empresa, con relación a sus actividades de comercialización de sus productos lo que se tendrá en cuenta en la capacitación, el cual debe comprender, en lo menor, de lo que seguidamente se presenta.

DIAGNOSTICO, básico para conocer la situación donde 
se encuentra la empresa, analizar por qué se llegó a esta situación. Estudiar cuáles son las tendencias actuales y cuáles son las necesidades del mercado.

PRONOSTICO, analizar las consecuencias de continuar la situación y en que desembocará en un plazo cercano. Qué hipótesis pueden ser formuladas con referencia a su futuro.

OBJETIVOS, dónde debe posicionarse realmente la organización en el futuro. Qué rentabilidad, beneficio o volumen de ventas se espera conseguir.

ESTRATEGIA, realizar un análisis para conocer cuál es el mejor alternativa para conseguir los objetivos, identificar cuáles son los factores críticos para alcanzar el éxito

GESTION, qué acciones específicas se deben adoptar. ¿Quién las debe aplicar? ¿Cuándo será el período más oportuno para llevar las a cabo?

CONTROL, que medidas deben adoptarse para detectar si el planteamiento se desarrolla según lo previsto.

Una presentación por etapas de actividades a desarrollar se menciona a continuación

PRIMERA ETAPA, deberá conocer la forma de identificar las necesidades del consumidor las cuales estarán vinculadas con las oportunidades para sus productos; identificadas deben aprovecharse o crearse las oportunidades. Proyectar la producción para satisfacer esas necesidades y la manera de informar a los consumidores de la disponibilidad del producto. Procedimientos a emplear para obtener la fidelidad de los clientes.

SEGUNDA ETAPA, utilizar procedimientos como: reuniones con los clientes, encuestas y otros. Cuál es la manera de procesar los datos externos (consumidores, competidores, normas legales, etc.) como emplear los datos internos (situación de la empresa, imagen ante los trabajadores, etc.) Explicar la forma como se selecciona el mercado indicando si es conveniente segmentarlo y de ser así posicionarse en el segmento elegido. Aplicar el marketing de productos de consumo en alimentación y de servicios si corresponde

TERCERA ETAPA, utilizar en forma constante estrategias para obtener beneficios: estrategias para el producto: diseño, marca, servicios adicionales; estrategias para el precio: formas y condiciones de pago, descuentos; estrategias para la plaza o promoción: canales, puntos de venta, transporte; estrategia de distribución extensiva; estrategia de distribución selectiva; y estrategia de distribución exclusiva.

Siendo estas aplicaciones de dinámica constante, deben realizarse cada cierto período el control del funcionamiento de lo programado, evaluar que se cumpla en los plazos que se han establecido los lanzamientos de nuevos productos, proyectar la utilización de otra marca, planificar servicios adicionales y otros.

\section{COSTOS: CLASIFICACION DE INSUMOS Y OBTENER COSTOS UNITARIOS}

\section{PRIMERA ETAPA}

Para el costo de un producto se debe tener en cuenta especificaciones o detalles así como procedimientos acordes con el producto por costear; progresivamente se explicarán y detallarán los conceptos para comprensión y utilización los cuales no serán los mismos en todos los casos, sin embargo la diferencia existente entre ellos no es significativa, por eso una presentación general permitirá, con adecuadas variables, utilizarlos en la mayor parte de los casos.

Algunas de las variables se mencionan para conocimiento general, sin establecer detalle de unidades monetarias ni unidades específicas, las cuales serán necesarias especificar cuando se aplique la capacitación, donde el costo tiene relación con la mayor pate; así se tiene: costo unitario, costo de materias primas, costo de mano de obra, costos indirectos de fabricación, costo de producción, costo de productos terminados, costo de productos en proceso, costos variables, costos fijos, costos históricos, costos predeterminados, costos de la producción equivalente, costos por órdenes de trabajo, costos por procesos, costos estimados, costos estándar y otros

La información cuantitativa será acumulada en la medida que se utilice, considerando los nombres usuales. Los importes totales, deben ser desagregados o unitarios para relacionarlos con los cronogramas de sembríos.

Las unidades de medida deben especificarse tales como: jornales, $\mathrm{m}^{3}$, litros, galones, kilogramos, etc.

La información, que después debe explicarse detalladamente, sería, en un inicio, similar a la que se presenta seguidamente, siendo los datos repetitivos por producto o campaña, es conveniente disponer de cuadros pre impresos con el detalle para que la tarea consista solo en anotar importes.

\section{SEGUNDA ETAPA}

La información se acumula cronológicamente incluyendo las inversiones significativas con sus especificaciones. Los importes totales se relacionan con el volumen de producción estimado para obtener los costos unitarios. Todas las inversiones permitirán realizar programaciones y aplicar técnicas administrativas como punto de equilibrio, costos conjuntos, entre otras.

Un posible bosquejo de la información seria como la que se presenta seguidamente, como la información es repetitiva es conveniente disponer de cuadros pre impresos con el detalle, en los que sólo se anotarán los importes. 
Tabla 1. Modelo para Costeo de Productos Agropecuarios.

\begin{tabular}{|c|c|c|c|c|c|}
\hline 10 Hectáreas & Arveja & 10 Hectáreas & Brócoli & 10 Hectáreas & Beterraga \\
\hline Semilla & $\mathrm{S} / 9800$ & Semilla & $\mathrm{S} / 1400$ & Semilla & $\mathrm{S} / 1800$ \\
\hline Siembra & $\mathrm{S} / 2400$ & Siembra & $\mathrm{S} / 7500$ & Siembra & $\mathrm{S} / 2400$ \\
\hline Riego & $\mathrm{S} / 11200$ & Riego & $\mathrm{S} / 12600$ & Riego & $\mathrm{S} / 14000$ \\
\hline Fertilizantes & $\mathrm{S} / 2800$ & Fertilizantes & $\mathrm{S} / 11800$ & Fertilizantes & $\mathrm{S} / 11800$ \\
\hline Pesticidas & $\mathrm{S} / 6000$ & Pesticidas & $\mathrm{S} / 1150$ & Pesticidas & $\mathrm{S} / 1150$ \\
\hline Aplicación pesticidas & $\mathrm{S} / 1800$ & Aplicación pesticidas & $\mathrm{S} / 1800$ & Aplicación pesticidas & $\mathrm{S} / 2100$ \\
\hline Aplicaciones fertilizantes & $\mathrm{S} / 900$ & Aplicaciones fertilizantes & $\mathrm{S} / 900$ & Aplicaciones fertilizantes & $\mathrm{S} / 720$ \\
\hline Labores culturales & $\mathrm{S} / 1500$ & Labores culturales & $\mathrm{S} / 3900$ & Labores culturales & $\mathrm{S} / 1200$ \\
\hline Deshierbo & $\mathrm{S} / 1500$ & Deshierbo & $\mathrm{S} / 1500$ & Deshierbo & $\mathrm{S} / 1200$ \\
\hline Espalderas & $\mathrm{S} / 12524$ & & & & \\
\hline \multirow[t]{2}{*}{ Cosechas } & $\mathrm{S} / 1500$ & Cosechas & $\mathrm{S} / 8400$ & Cosechas & S/ 6000 \\
\hline & S/ 51924 & & $\mathrm{~S} / 50950$ & & S/ 58570 \\
\hline
\end{tabular}

Tabla 2. Modelo para obtener el costo unitario en productos agrarios. Holantao 5 Hectáreas Producción Kilogramos 55.000 .

\begin{tabular}{cccccc}
\hline & Unidad & Cantidad & Costo Unitario & Importe & $\begin{array}{c}\text { Costo Variable } \\
\text { unitario }\end{array}$ \\
\hline Preparación terreno & Máq. & 5 & $\mathrm{~S} / 300.00$ & $\mathrm{~S} / 1500.00$ & 0.027273 \\
Siembra & Jornal & 30 & $\mathrm{~S} / 20.00$ & $\mathrm{~S} / 600.00$ & 0.010909 \\
Semillas & $\mathrm{Kg}$. & 180 & $\mathrm{~S} / 8.00$ & $\mathrm{~S} / 1440.00$ & 0.026182 \\
Pesticidas & & & $\mathrm{S} / 650.00$ & $\mathrm{~S} / 3250.00$ & 0.059091 \\
Aplicación pesticidas & Jornal & 28 & $\mathrm{~S} / 20.00$ & $\mathrm{~S} / 560.00$ & 0.010182 \\
Cosecha & Jornal & 190 & $\mathrm{~S} / 20.00$ & $\mathrm{~S} / 3800.00$ & 0.069091 \\
Labores culturales & Jornal & 30 & $\mathrm{~S} / 20.00$ & $\mathrm{~S} / 600.00$ & 0.010909 \\
Deshierbo & Jornal & 35 & $\mathrm{~S} / 20.00$ & $\mathrm{~S} / 700.00$ & 0.012727 \\
Agua & $\mathrm{M}^{3}$ & 38000 & $\mathrm{~S} / 0.15$ & $\mathrm{~S} / 5700.00$ & 0.103636 \\
Fertilizante & - & - & - & $\mathrm{S} / 1500.00$ & 0.027273 \\
Aplicación fertilizante & Jornal & 16 & $\mathrm{~S} / 20.00$ & $\mathrm{~S} / 320.00$ & 0.005818 \\
Espalderas & - & - & $\mathrm{S} / 1300.00$ & $\mathrm{~S} / 6500.00$ & 0.118182 \\
& & & $\mathrm{~S} / 26470.00$ & 0.481273 \\
\hline
\end{tabular}

\section{TERCERA ETAPA}

Se aplicarán algunas herramientas de administración como la siguiente:

Tabla 3. Modelo para control de las actividades en procesos agrarios.

\begin{tabular}{|c|c|}
\hline PAPA & ICA \\
\hline $\begin{array}{l}\text { 1Ha } \quad 45000 \mathrm{~kg} . \\
\text { Siembra: Marzo } \\
\text { Cosecha: Julio }\end{array}$ & $\begin{array}{c}\text { Cultivo de rotación } \\
\text { Tiempo: } 105 / 120 \text { días }\end{array}$ \\
\hline $\begin{array}{l}\text { PREPARACION DE } \\
\text { TERRENO }\end{array}$ & $\begin{array}{l}\text { - } \quad \text { Riego machaco } \\
\text { Tractor alquiler una hora: } \\
\text { a. Aradura máxima } 2 \text { vueltas al campo. } \\
\text { b. Surcos (abonos, estiércol). } \\
\text { - Diez jornales ( } 8 \text { horas), en un día. }\end{array}$ \\
\hline SIEMBRA & $\begin{array}{l}\text { - Son } 10 \text { jornales } \\
\text { - } \quad \text { Seleccionar semilla: siembran. } \\
\text { - } \quad \text { Alimentos y reunión opcional. }\end{array}$ \\
\hline INSPECCIÓN & $\begin{array}{l}\text { - } \quad \text { Riegos cada } 15 \text { días gravedad, aspersión, goteo. } \\
\text { - } \quad \text { Terreno: franco, arenoso, salino. } \\
\text { - } \quad \text { Control constante plagas: gusanos mosca minadora. } \\
\text { - Productos químicos contra plagas y/o enfermedades. }\end{array}$ \\
\hline
\end{tabular}


Continuación de Tabla 3

\begin{tabular}{|c|c|}
\hline APORQUE & $\begin{array}{l}\text { - } \quad \text { Refuerzo a la planta para que no se caiga por crecimiento. } \\
\text { - } \quad \text { Verificación del control de humedad. } \\
\text { - Cinco jornales, en un día. }\end{array}$ \\
\hline INSPECCIÓN & $\begin{array}{l}\text { - } \quad \text { Riegos cada } 15 \text { días gravedad, aspersión, goteo. } \\
\text { - } \quad \text { Terreno: franco, arenoso, salino. } \\
\text { - } \quad \text { Control constante plagas: gusanos mosca minadora. } \\
\text { - Productos químicos contra plagas y/o enfermedades. }\end{array}$ \\
\hline COSECHA & $\begin{array}{ll}\text { - } & \text { Tractor } 1 \text { día dos vueltas. } \\
\text { - } & \text { Doce (12) jornales, en un día. } \\
\text { - } & \text { Recoger y llevar a punto acopio, clasifica y ensaque. } \\
\text { - } & \text { Recoger seleccionado (clasificación) llevar a acopio. } \\
\text { - Semillas, alimento ganado. } & \text { Sacos y selladora. } \\
\text { - Comida y reunión opcional. }\end{array}$ \\
\hline VENTA Y/O POR VENDER & - Almacén, mercados, acopio, mayorista y transporte. \\
\hline OTROS & - Alquiler de área, almacén, ganado y herramientas. \\
\hline
\end{tabular}

\section{Conclusiones}

La capacitación a pequeños productores agrarios, familiares y relacionados en producción agraria y administración aplicada al sector permitirá que mejoren su calidad de vida así como el enseñarles la ventaja de utilizar tecnologías limpias, el acceso a herramientas administrativas permitirá modificar el modelo actual de subsistencia. Si se realiza la capacitación en tres etapas; este es un procedimiento inicial después, si hay menos o más capacitaciones, dependerá de los avances tecnológicos y del grado conocimiento que tengan. Tanto los investigadores como periodistas, empresarios y otros coinciden en que la capacitación del área rural será altamente beneficiosa por ello se presenta un procedimiento para aplicación.

\section{Literatura citada}

Agronoticias. 2014. "Frente al año internacional de la agricultura familiar y la COP 20".Agronoticias.NO.396: Pág 2.

Bagum, M., Saikia, M. 2013. Effect of irrigation on mulching on growth and yield attributes of popato. Agricultural Research Communication Centre 34(1)7678.

Castañeda, P, E. 2013. "Establecer módulos demostrativos con grupos de 25 a 30 familias afines". Agronoticias. no.393: Pág 18-19.

Cuellar, T, D. 2013. Fortalecimiento de la cadena de valor del cacao criollo cono certificación orgánico solidario en agricultores de la cac_pangoa. Ingeniero en gestión empresarial. Lima, pe. Universidad Nacional Agraria La Molina. pág 73.

De Lucía, B.; Cristiano, G.; Vecchietti, l.; Rea, E. y Russo, G. 2013. Nursery $>$ Growing Media: Agronomic and Environmental Quality Asessment of Sewage Sludge-Based compost.environmental soil science article id 565139, 10 pages.
Huamán A, J. 2013. Fortalecimiento de la cadena productiva de palto de la asociación agromashacat, provincia de celendín - región Cajamarca. mg sc. Lima, pe. Universidad nacional agraria "la molina". pag 107.

Lacki, P. 2006. El verdadero libro de los pobres rurales. Brasil. consultado 6 de octubre del 2013. disponible en HTTP://WWW.POLANLACKI.COM.BR

Medina, M, f. 2013. Gestión empresarial de micro y pequeñas empresas agro procesadoras. . mg sc. Lima, pe. Universidad Nacional Agraria "la molina". pag 48.

Narth, A.; Wilkinson, S. 2013. Can we build better compost? use of waste drywall to enhance plant growth on reclamation sites. Journal Of Environmental Management. nov. 2013, vol. 129, p 503-509. 7p.

Varela, V, R. 2008. Innovación empresarial. Arte y ciencia en la creación de empresas. Editora Castillo m. Tercera edición. Bogotá d.c., co. Pearson Educación de Colombia ltda no. pág125 - 142 .

Webb, Agricultura de maceta. El comercio 26/08/2013. 